\title{
La gobernanza mundial y el derecho mundial en un mundo fragmentado*
}

Dieter Senghaas ${ }^{* *}$

En una 'Breve historia del mundo globalizado del siglo XXI', título del libro exitoso aparecido en 2005 y traducido a varios idiomas, Thomas L. Friedman columnista del New York Times y autor de bestsellers sostiene que el mundo es plano ${ }^{1}$. En efecto, algún elementos del mundo globalizante confirman en cierta forma esta perspectiva, como se comprueba en el rol y función de la Internet, en las cadenas globales de valor vía "outsourcing» o subcontratación, «offshoring» e «insourcing», en la planificación digital de los ritmos de trabajo, así como en otros procesos que son objeto de organización global.

Publicado originalmente en alemán en Vereinte Nationen 1/2009, pp. 11-16.

** Catedrático del Institut fue Interkulturelle und Internationale Studien, Universidad de Bremen.

1 Thomas L. Friedman, (2005) La Tierra es Plana: Breve historia del mundo globalizado del siglo XXI, Martínez Roca, Barcelona 2005.
Sin embargo, esta perspectiva, algo exagerada, resulta demasiado para describir adecuadamente o realizar un examen analítico del mundo real existente. Ello porque el mundo actual se caracteriza por grandes disparidades y situaciones problemáticas globales del más diverso orden.

\section{UN MUNDO FRAGMENTADO}

La estructura macroeconómica mundial se caracteriza por una extremada jerarquización y estratificación. Existe un vacío entre un centro de gravitación, que se distingue por la interconexión simétrica densa, y el «resto del mundo». Este centro de gravitación, constituido por el espacio que abarca la Organización para la Cooperación y el Desarrollo Económicos (OCDE), que con una participación del $15 \%$ de la población mundial contribuye al $75 \%$ del producto bruto interno 
mundial, domina en todos los aspectos. En cambio, la estructura del resto del mundo -que representa la mayor parte de la población mundial- se distingue por una interdependencia asimétrica que se traduce en una dependencia estructural frente a este centro de gravitación. Hasta ahora no hay otro centro de poder colectivo y coordinado que se le compare y se le oponga, ni siquiera a nivel regional. No obstante, se ha traído a discusión la aparición del llamado Grupo-BRIC, formado por Brasil, Rusia, India y China, como posible constelación de poder emergente que pudiese hacerle contrapeso al predominio de Estados Unidos y al espacio de la OCDE en su conjunto, así como a las organizaciones internacionales en que influyen decisivamente. Pero en vista de las realidades políticas mundiales prevalecientes aún, se trata aquí más bien de un deseo y no de un análisis serio.

En esta estructura mundial se perciben tanto a nivel global como en las sociedades en particular procesos de movilidad social ascendente (vgr. Asia del Este) y movilidad social descendente (vgr. África subsahariana). Mas estos adquieren grandes dimensiones cuando, al darse simultáneamente movilidad social ascendente y descendente, se llega a un vacío o disparidad absoluta entre ellas, como sucede entre la OCDE o Asia del Este en que se da la primera, y la África subsahariana, que se caracteriza por la segunda. Pero también en el caso individual de sociedades de movilidad social ascendente, como China, se presentan fragmentaciones internas de magnitud relativa o absoluta, que por lo general son de carácter geográfico o social. Incluso el mismo espacio de la OCDE está expuesto a las tendencias de fragmentación, aunque en medida moderada debido principalmente al éxito de una competencia desplazante en esferas productivas situadas por debajo de los sectores punta, ocasionada por países emergentes de movilidad social ascendente. En tales sociedades se va formando entonces un "polo marginal» de personas que en el mercado de trabajo ya no son productores ni consumidores, característica que ha sido siempre común en los llamados países del Tercer Mundo.

El diagnóstico sobre América Latina en los años sesenta y setenta, producto del debate acerca de la «dependencia», que revela la existencia de una fragmentación interna creciente producto de la integración en una economía mundial de estructura asimétrica (llamada «heterogeneidad estructural»), se ha convertido en un fenómeno mundial, con la característica particular de que en esta oportunidad es escalonado.

\section{CONTROVERSIA EN LA GOBERNANZA MUNDIAL}

¿Qué consecuencias acarrea esta macroestructura para la gobernanza mundial y las ideas programáticas sobre el orden mundial? ${ }^{2} \mathrm{Al}$ respecto, cabe distinguir cuatro modelos:

2 Para discusiones posteriores vid. también Volker Rittberger, «Weltregieren: Was 
Dieter SenghaAs • La gobernanza mundial y el derecho mundial...

\section{Imperio/hegemonía}

Una estructura jerarquizada y graduada, que muestra relaciones densas de los actores en su cúspide y una gran fragmentación de ellos en su parte inferior, provoca efectivamente ideas programáticas imperiales o hegemónicas sobre el orden mundial. Pero a diferencia de tiempos pasados, hoy estas ideas se asemejan más a fantasías irracionales de poder que a conceptos operativos realistas. A pesar de ello, ha habido intentos de poner en práctica una política de tal orientación, como se aprecia en el caso de Estados Unidos con la consigna de la «unipolaridad». Su fracaso es inevitable. La razón principal es que actualmente la movilización social de sociedades por separado y su consiguiente politización global -precisamente en la zona de fragmentación-, están demasiado avanzadas como para dar pie a una política imperial o hegemónica de este tipo que, al menos transitoriamente en tiempos pasados fue imaginable y fiable. Con todo, siguen existiendo "cabezas de puente» que son indispensables para una política imperial y hegemónica y que actúan a nivel local -como sucede con el régimen de Hosni Mubarak en Egipto para la política de Estados Unidos y Occidente. Pero estas «cabezas de

kann es leisten? Was muss es leisten? « en Hans Küng /Dieter Senghaas (eds.) (2003), Friedenspolitik, Munich, Hauser, pp. 177208. En esta aportación se discuten tres modelos de orden mundial: Estado mundial, hegemonía y coordinación política horizontal. puente» que se comprometen de esta manera se convierten también en blanco de una creciente resistencia política local. En la mayoría de los casos, solo se mantienen en el poder por un tiempo y gracias a una política de clientelismo financiada por el extranjero, sumada a un aumento de la represión, que agrava la situación política del lugar.

\section{Concierto multipolar de las potencias}

Otra idea sobre el orden mundial, que se entiende como realismo político, ve una posible solución al problema en una constelación de grandes potencias, que a su vez estarían formadas por potencias líderes regionales. Concepciones de este tipo consideran a Estados Unidos, la Unión Europea y los Estados del grupo BRIC como los guardianes privilegiados del orden político mundial. Pero estas potencias líderes regionales también están inmersas en la misma problemática en el sentido de que son aspirantes potenciales a una política imperial mundial, lo que significa recurrir a prácticas de dominación sobre Estados y pueblos que generalmente se encuentran en situaciones de cambio político, social y cultural, sin que tenga que ser más rentable hacerlo desde afuera. A ello se suma el hecho de que la conformación de nuevas constelaciones de poder ha provocado -por lo menos en la historia contemporánea actual- una problemática llamada de transición del poder, esto es, una cons- 
telación conflictiva entre una potencia anteriormente líder pero actualmente en vías de declinación y una nueva potencia en acenso que tiene posibilidades de acceder al poder ${ }^{3}$; constelación que se está formando principalmente entre Estados Unidos y China y que es materia de intenso debate en ambos países. De momento se hace difícil anticipar una respuesta a la cuestión de si en la práctica esta problemática de crisis hegemónica adoptará realmente la forma de una relación política mundial conflictiva y virulenta o si el resultado de tal desplazamiento del poder de relevancia política mundial será un proceso atenuado de formación de un acuerdo entre ambas o más potencias que desembocará en un concierto multipolar de potencias.

\section{El orden de las Naciones Unidas}

Una tercera idea programática del orden mundial centra la atención en el sistema de las Naciones Unidas. En realidad, ella debería mencionarse en primer lugar, puesto que hoy no hay duda que de acuerdo con el derecho internacional público, las Naciones Unidas constituyen el marco regulatorio obligatorio de la vida internacional. El sistema ha sido capaz de ponerse a la altura de los tiempos y ha obtenido mayores logros que las ideas programáticas mencionadas anteriormente, pues

Al respecto es fundamental la obra de Robert Gilpin, (1981) War and Change in World Politics, Nueva York, Cambridge UP. tiene orientación universal y cuenta con múltiples subsistemas regionales y funcionales.

En su calidad de marco regulatorio mundial, las Naciones Unidas se basan en principios tradicionales, nuevos y en proceso de formación. Entre los primeros cabe mencionar la soberanía y la no intervención en los asuntos internos, y entre los nuevos -frente al derecho internacional público clásico- la prohibición general del recurso a la fuerza con sus modalidades derivadas de la seguridad colectiva. En lo referente al surgimiento de situaciones problemáticas no previstas en 1945 se ha ido avanzando hacia una interpretación extensiva de conceptos elaborados originalmente de forma restrictiva y nociones operativas, sobre todo respecto de lo que se entiende o debe entenderse como «amenaza a la paz» (Art. 39 de la Carta de Naciones Unidas) en caso de que se produzca un conflicto armado o de que haya peligro de que pueda producirse. Estas nuevas interpretaciones restringen los derechos de soberanía de los miembros de las Naciones Unidas, aunque no siempre de forma fiable.

Si las decisiones y actuaciones de las Naciones Unidas fueran coherentes y sostenidas en el sentido anotado, garantizándose con ello su fiabilidad, cabría prever el tránsito de un derecho internacional público de corte tradicional a un derecho mundial emergente, operable a nivel global y de carácter preeminente. Pero existe una situación que se opone tajantemente a este tránsito imaginable e incluso deseable: el rol problemático 
Dieter SenghaAs • La gobernanza mundial y el derecho mundial...

del Consejo de Seguridad. Este órgano es reflejo de una constelación clásica de poder de las potencias; sus miembros pueden ejercer a voluntad una política de intereses nacionales de facto $y$, teniendo en cuenta su estructura, también de jure. Así pues, sus decisiones se toman mayormente a la carta, a la par que tienen carácter jurídico puesto que si bien queda por aclarar su alcance, de acuerdo con el artículo 25 de la Carta son obligatorias y se elaboran o concluyen sin un procedimiento legislativo y sin prever su control jurídico -aparte de que la competencia obligatoria de instituciones como la Corte Internacional de Justicia no incluye tal examen jurídico. Esto hace que nos encontremos en presencia de un órgano de las Naciones Unidas de corte neoabsolutista ${ }^{4}$.

Por lo general, se responde a las críticas a esta situación con dos argumentos. En primer lugar, se sostiene que el perfil que se dio en la Carta a este órgano central de las Naciones Unidas, que constituiría una especie de comité central, fue deliberado, puesto que sin esta conceptualización no se habría podido crear. En segundo lugar, se argumenta que si no se hubiera dado el poder de veto a las potencias y al Consejo de Seguridad en su conjunto, la organización habría tenido menos capacidad de acción de la que tiene

4 Vease. Senghaas Dieter, (2004) Zum irdischen Frieden. Erkenntnisse und Vermutungen, Frankfurt a.M. Suhrkamp, p. 55 ss; Senghaas Dieter, (2007) On Perpetual Peace. A Timely Assessment, Nueva York /Oxford, Berghahn Books. actualmente. En todo caso, habría que examinar más a fondo tanto este argumento como la tesis contraria, que sostiene que la estructura institucional del Consejo de Seguridad, análoga a la del Estado de derecho, le darían reconocimiento y legitimidad, y por extensión a las Naciones Unidas, lo que al mismo tiempo se traduciría en una mayor disposición a movilizar recursos necesarios.

Con todo ello, la problemática recién descrita -ausencia de principios análogos a los del Estado de derecho como son los del debido proceso y del imperio del derecho - viene mucho más al caso que discutir sobre el aumento de número de los miembros del Consejo de Seguridad, su división en permanentes y no permanentes y con o sin derecho de veto. Ello porque la ampliación del tamaño de este órgano superior no atenuaría ni menos alteraría sustancialmente su carácter neoabsolutista.

\section{Sistemas reguladores globales}

Una cuarta idea programática del orden mundial se vincula actualmente al concepto de «gobernanza mundial», que se puede apreciar sobre todo en los debates de la ciencia política sobre las relaciones internacionales. En el marco de ellos se ha formulado este concepto de forma relativamente amplia: una gobernanza mundial que se manifiesta en la creación institucional de esferas problemáticas globales relacionadas principalmente con campos políticos específicos. 
Así pues, la «gobernanza mundial» se aprecia en un amplio espectro de sistemas reguladores internacionales o regímenes de las más distintas características. Por un lado, estos tienen perfiles de índole normativa, institucional y procedimental relativamente densos, de alta efectividad, que llegan a ser aceptables pero cuya legitimidad es cada vez más cuestionable. Por el otro, incluyen proyectos de "gobernanza mundial» deseables, es decir, de estructuras que en el sentido estricto del concepto no existen todavía pero que son enunciados en declaraciones e ideas programáticas sobre una estructura del orden mundial deseable en un campo político específico. El primero de ellos puede verse en la Organización Mundial del Comercio (OMC); el último, en los esfuerzos por coordinar o al menos concertar una cooperación para el desarrollo mediante sistemas reguladores internacionales a nivel diplomático y en medio de una multiplicidad de actores internacionales, estatales y privados ${ }^{5}$.

\section{SISTEMAS REGULADORES:}

\section{AMPLIOS Y DÉBILES}

Por regla general y a nivel global, los sistemas que regulan un campo político específico o los acuerdos de

Sobre esta problemática vease. Breitmeier Helmut, Michèle Roth y Dieter Senghaas (Eds.), (2009) Sektorale Weltordnungspolitik. Effektiv, gerecht und demokratisch?, Baden-Baden, Nomos (con casos de estudio sobre diez sistemas regulativos globales de distinta naturaleza). gobernanza mundial no pueden sustraerse a las disparidades a que da lugar la macroestructura mundial, si bien algunos de estos acuerdos institucionales o cuasi-institucionales apuntan a contrarrestar estas fragmentaciones. A manera de ejemplo cabría mencionar la marcada fragmentación del poder global que se aprecia en el régimen de no proliferación nuclear. Creado con la intención de impedir la propagación de armas nucleares y orientado al desarme nuclear de las potencias nucleares, conduce más bien a la proliferación nuclear y no a un mundo sin esta clase de armas debido al desgaste progresivo de su legitimidad como resultado del no-desarme de las potencias nucleares.

Muy diferente es el régimen del comercio mundial, que a partir del fin de la Segunda Guerra Mundial ha ido adquiriendo paulatinamente mayor densidad, a la vez respecto de los fines -abarcar más esferas para el libre comercio-, y de la estructura institucional del sistema, la OMC, con su mecanismo institucionalizado de solución de disputas. Este mecanismo ya no se basa en procedimientos de negociación políticamente motivados y de cumplimiento por vía diplomática como se dio en su organización predecesora, el GATT, sino que consiste en un procedimiento cuasijurídico. Sin embargo, este sistema regulador, que se asimila cada vez más a un subsistema global sólido tampoco está exento de problemas: parte del supuesto de que el libre comercio per se es el promotor del bienestar y, sobre todo, del desarrollo, como en tiempos del 
Dieter SenghaAs • La gobernanza mundial y el derecho mundial...

GATT. Consecuentemente, se sostiene que el libre comercio contribuye a reducir las disparidades socioeconómicas que existen al interior de las sociedades, y sobre todo entre las sociedades desarrolladas y menos desarrolladas. Pero una sujeción incondicional y por ende dogmática al libre comercio impide darse cuenta de las condiciones que deben darse en los planos internacional y local para que pueda producir los efectos que el sistema abstractamente supone que se dan en toda circunstancia o que pueden lograrse. En este contexto, las experiencias del desarrollo histórico europeo y, en el último tiempo, de los países emergentes de Asia del Este son de fundamental importancia. El éxito con que se desarrollaron estos espacios económicos -incluidos América del Norte y Oceanía- no se orientó simple y llanamente al libre comercio sino a un desarrollo à la Friedrich List: integración selectiva en la economía mundial con un desenganche selectivo temporal, a fin de desarrollar el propio mercado interno con eficiencia en todos los sectores y a largo plazo, al libre comercio ${ }^{6}$.

Para que la estructura de la economía mundial sea equilibrada convendría que el régimen regulador de la OMC y los convenios sobre derecho laboral y las normas sociales que han alcanzado considerable perfección y se encuentran

6 Friedrich List, (2008), Das internationale System der politischen Ökonomie (1841), Baden-Baden. Al respecto, ver Dieter Senghaas, «Wege aus der Armut. Was uns Friedrich List und die Entwicklungsgeschichte lehren ", Blätter für deutsche und internationale Politik, 12/2008, pp. 79-95. principalmente en los convenios de la Organizació Internacional del Trabajo (OIT) estuviesen directamente relacionados. De esta manera, se superaría el recorrido infructuoso de cada uno de ellos por separado. En este recorrido se percibe por un lado la orientación neoliberal del sistema que rige predominantemente el comercio mundial y, por el otro, la debilidad política relativa de los proyectos globales de derecho laboral que promueve la OIT.

Asimismo, en vista de la última crisis financiera internacional y de la sorprendente falta de regulación y de control de las transacciones financieras internacionales se hace necesario someterlas a una regulación cautelosa mediante instancias públicas, a la par que se torna indispensable evitar que los bancos evadan «legalmente», convenios como Basilea I y II -que prevén algunas modalidades de autoregulación, como la fijación de cuotas al capital propio en el otorgamiento de créditos. El fortalecimiento de la regulación de este sector mediante uno o más sistemas reguladores podría y debería contribuir a generar transparencia y obligar a los actores e instituciones centrales del mundo financiero internacional a rendir cuentas, contrarrestándose así imperfecciones de la economía nacional y global producto de una regulación inadecuada. El hecho de que hasta ahora prácticamente no se han aplicado normas en el sentido indicado pone de manifiesto las relaciones reales de poder que existen a nivel mundial, que resultan en un predominio de ideas progra- 
máticas neoliberales. En este contexto es sorprendente notar que el comercio internacional de productos y servicios -regulado de diversa forma por la OMC y acuerdos regionales y bilaterales-, asciende aproximadamente a 12 billones de dólares de Estados Unidos al año (2006), mientras que en los centros financieros globales las transacciones se elevan a unos 3 billones de dólares por día bursátil, ello sin regulación alguna, lo que en 250 días de actividad bursátil se eleva aproximadamente a 750 billones de dólares, cifra 60 veces superior a la del comercio mundial anual de productos y servicios.

Este espacio financiero internacional, que por voluntad política continúa estando insuficientemente regulado, contrasta ostensiblemente con una multiplicidad de sistemas reguladores o convenios globales, regionales, subregionales y bilaterales como los existentes en el área medioambiental, cuyo espectro se extiende desde la Convención Marco de las Naciones Unidas sobre el Cambio Climático de 1992 hasta los distintos convenios para la prevención de la contaminación de los ríos y los mares, para cuya conclusión y aplicación en muchos casos cumplen un papel importante las organizaciones no gubernamentales. Cabe también mencionar la existencia de una diversidad de redes de contactos o comunidades virtuales con una pluralidad de actores -incluyendo fundaciones con fuerte financiación-, como signo característico de una "gobernanza mundial de la salud « paralela a la existencia de la Organización Mundial de la Salud (OMS); constatándose en este caso que de existir discrepancia entre ella y los actores privados en la asignación de recursos materiales, se inclinaría resueltamente en favor de las actividades de las redes de contacto. Visto desde un ángulo conceptual, este ejemplo nos remite al tránsito hacia formas de «autoregulación regulada», esto es, una «gobernanza mundial» concebida como interacción entre el Estado -que actúa de fondo- y una organización autónoma privada, como se observa también en la gobernanza de la red en el marco de la Corporación de Internet para la Asignación de Nombres y Números (ICANN). Por último, cabe mencionar la adopción autónoma o autogestionaria de normas internacionales obligatorias y su implementación en el marco de un derecho privado mundial (Lex Mercatoria, Lex Sportiva, Lex Informatica), por parte de empresas y asociaciones u organizaciones de carácter privado.

Todo este amplio espectro de acuerdos -desde convenios como el de no proliferación nuclear hasta acuerdos privados de carácter jurídico globalsurgen de la necesidad de coordinar y solucionar los problemas en estos campos políticos o problemáticos, al ser evidente que rebasan la capacidad y competencia de los Estados y actores para resolverlos individualmente. 
Dieter SenghaAs • La gobernanza mundial y el derecho mundial...

Sistemas Reguladores COMO RESULTADO DE DISCURSOS

\section{GLOBALES}

En este contexto debe resaltarse la importancia que han tenido en años pasados, y seguirán teniendo en el futuro, los discursos públicos o debates de políticos, representantes de intereses y científicos (estos últimos en el marco de las llamadas «comunidades epistémicas»), en temas como la reorientación política mundial hacia la responsabilidad de proteger que corresponde a la comunidad internacional en casos de violación grave de los patrones internacionales de conducta ampliamente aceptados y de las situaciones de emergencia de la población como resultado de su violación («responsibility to protect») 7 . Se trata aquí de un proceso político y programático que ha aparecido hace unos años y goza de resonancia mundial considerable, pero que no ha llegado a constituir un régimen estable garantizado institucionalmente, ni qué decir de su implementación en casos concretos a partir de normas obligatorias.

Sin embargo, tal sistema regulador, en sí deseable, presupondría una casuística de intervenciones legítimas de aceptación general, según la cual se actuaría de la misma forma para casos iguales dentro del marco de un procedimiento análogo al del Estado de derecho, hecho que implicaría una reforma

Al respecto, véase la excelente monografía de Detlev Wolter (2007), A United Nations for the 21st. From Reaction to Prevenion, Baden-Baden, Nomos.. sustancial del Consejo de Seguridad. Mas la comunidad internacional está aún muy lejos de llegar a tal régimen y probablemente no lo llegue a alcanzar, pero un paso importante digno de destacar es el discurso público.

Conflicto sobre el derecho mundial La controversia política y científica actual sobre la responsabilidad de proteger de la comunidad internacional -que también se seguirá observando en un tiempo previsible- revela la forma usual en que se concluyen los diversos tipos de regímenes internacionales o acuerdos de gobernanza mundial. Ellos son en general el resultado de una ardua historia de disputas, en la cual los defensores de ideas programáticas nuevas y visionarias enfrentan la resistencia de los partidarios del statu quo, con la diferencia de que en el pasado los representantes de ambas posiciones generalmente provenían de instituciones estatales, mientras que ahora el componente civil de las organizaciones no gubernamentales y asociaciones económicas nacionales e internacionales juega un papel relevante en tales controversias. Esto queda más de manifiesto en el desarrollo histórico de los debates sobre el contenido y alcance de los derechos humanos. Partiendo de la cuestión fundamental del valor jurídico de estos en el derecho internacional público tradicional y del valor que deben tener los derechos humanos de segunda, tercera y otras generaciones, cabe preguntarse si se ha superado realmente la posición del individuo como sujeto no autónomo supuesta hasta entonces 
por la dogmática en general y válida en el derecho internacional público. En comparación con el pasado ¿se ha convertido el individuo o, por lo menos, se está convirtiendo verdaderamente en un sujeto inmediato del derecho internacional público -derecho que en tal sentido llevaría mejor el nombre de derecho mundial? ¿Está el derecho internacional público tradicional dispuesto a dejar espacio para principios de un derecho mundial como los mencionados arriba ${ }^{8}$ ?

No se puede responder a esta clase de cuestiones con una afirmación o negación categóricas. La controversia entre las respuestas políticas deseables y las jurídicas moderadas se mantendrá por años y quizás por decenios, debido a que las relaciones jurídicas que derivan del mundo real y a que se ha aludido más arriba son de tal complejidad que exigen interpretación, existiendo muchas zonas intermedias 9

En este contexto, un acontecimiento digno de mencionar pero no necesaria-

$8 \quad$ Vease en especial el estudio monumental e innovativo de Angelika EmmerichFritsche (2007), Vom Völkerrecht zum Weltrecht, Berlin,Duncker y Humblot.

9 Para aportaciones interesantes en este sentido vid. von Schorlemer, Sabinde (2006), «Imperative eines künftigen Friedensvölkerrechts « en Dieter Senghaas (ed.), Konstruktiver Pazifismus im 21. Jahrhundert, Viena. LIT,pp 33-66; Habermas, Jurgen (2005), "Eine politische Verfassung für die pluralistische Weltgesellschaft? " en Jürgen Habermas, Zwischen Naturalismus und Religion. Philosophische Aufsätze, Frankfurt a.M, Suhrkamp, pp.. 324-365. mente representativo ${ }^{10}$ es la creación de la Corte Penal Internacional, con la forma especial en que se estableció y la responsabilidad penal individual de los individuos en el derecho internacional. Sin embargo, el procedimiento orientado al principio de subsidiaridad es cuestionable y más aún, Estados importantes como Estados Unidos rechazan este tribunal. Con todo, los Estados signatarios del Estatuto de Roma han otorgado jurisdicción a la Corte para los crímenes más graves, a saber, genocidio, crímenes de lesa humanidad y crímenes de guerra. Aquí se observa un cambio de paradigma de un derecho internacional público a un derecho mundial pero de forma reducida, en vista de las limitaciones examinadas. Anterior y más progresivo en su alcance, y por tanto, más trascendental, ha sido el surgimiento de un derecho imperativo (ius cogens) y de obligaciones erga omnes, es decir, de principios que teniendo como destinatario al Estado (soberanía, prohibición del recurso a la fuerza, derecho de la autodeterminación de los pueblos), están predominantemente orientados a la protección de los individuos (prohibición del genocidio, esclavitud, discriminación racial y tortura; crímen de lesa humanidad, entre otros).

Desarrollos de este tipo -independientemente de que se consideren procesos de formación de un derecho

10 Véase Deitelhoff, Nicole (2006), Überzeugung in der Politik. Gründzüge einer Diskurstheorie internationalen Reigerens, Frankfurt a.M., Suhrkamp.. 
Dieter SenghaAs • La gobernanza mundial y el derecho mundial...

mundial interno (Weltinnenrecht), derecho mundial (Weltrecht) o derecho de la humanidad, e independientemente de la importancia jurídica operativa que obtengan a nivel supremo (tratándose del ius cogens) o en un ámbito sectorial- se caracterizan por tendencias o corrientes de aceptación y de reticencia, e incluso rechazo. Ello dificulta en si emitir juicios sobre la situación ${ }^{11}$. Pero la mayor dificultad radica en la macroestructura global mencionada al comienzo. Pues los avances hacia un derecho mundial o cuasimundial presuponen una estatalidad sólida e incluso una forma democrática que satisfaga los principios del Estado de derecho, presupuesto al que también está sujeto el derecho internacional público tradicional en su variante de derecho de coordinación y de cooperación. Pero precisamente este requerimiento riguroso fracasa en numerosos lugares y no solamente en los Estados fallidos o en peligro de fracasar. A ello se agrega el hecho de que Estados constitucionales democráticos como Estados Unidos no siempre se consideran los portavoces para la aceptación e implementación de un derecho mundial.

Estas situaciones contribuyen a que en los próximos decenios, concepciones del orden mundial de gran envergadura - como la idea programática de una democracia cosmopolita, una civitas mundial o república de las repúblicas- pero desconocedoras de las disparidades

11 Al respecto, ver Kreide,. Regina y Andreas Niederberger (eds.)(2008), Transnationale Verrechtlichung, Frankfurt a.M., Campus. globales lamentablemente solo seguirán teniendo una relevancia abstracta. Con ello no se intenta fundamentar objeciones a discursos particularmente de orientación filosófica sobre la materia, sino que se advierte respecto de un escepticismo moderado en vista de la situación mundial real.

\section{ACumulación de la pREsión DE LOS PROBLEMAS GLOBALES}

Los discursos a que nos hemos referido adquirirán importancia programática y práctico-operativa cuando se hagan más evidentes las acciones restrictivas resultantes de las situaciones problemáticas mundiales identificadas, que tienden a acentuarse en los próximos años ${ }^{12}$. Pertenecen a esta clase de situaciones:

- La problemática medioambiental global (cambio climático, destrucción de la capa de ozono, pérdida de la diversidad biológica, pérdida de suelos fértiles, destrucción de las selvas tropicales, crisis global del agua, interacción de daños ecológicos condicionados por la riqueza y la pobreza);

- Posible escasez de materias primas y energía con su correspondiente modificación de los precios;

$12 \mathrm{Al}$ respecto, véase Ferdowsi, Mir A. (ed.) (2007), Weltprobleme, Munich, Bayerische Landeszentrale für politische Bildungsarbeit; Ferdowsi; Mir A. (ed.) (2009), Internationale Politik als Überlebensstrategie, Munich, Bayerische Landeszentrale für politische Bildungsarbeit.. 
- Propagación de armas de destrucción masiva;

- Déficits de regulación o ausencia de sistemas reguladores de alcance global, de enormes consecuencias a nivel mundial (como se ha observado con claridad desde 2007 en los mercados financieros internacionales y en la economía real);

- Epidemias globales que se transmiten cada vez con mayor velocidad;

- Crisis de desarrollo crónicas junto al peligro del colapso de Estados y el surgimiento de movimientos fundamentalistas y terroristas de alcance local, regional, e incluso global y

- Movimientos migratorios consecuencia de cambios demográficos de distinta naturaleza en diferentes partes del mundo, pero sobre todo a consecuencia de daños socio-económicos y políticos en regiones pobres con altos índices de crecimiento demográfico.

Cada una de estas situaciones problemáticas y con mayor razón la suma de ellas puede conducir a una sobrecarga de los sistemas de conocimiento y de formación de la voluntad y decisión en los más distintos niveles del orden mundial, por lo cual no es improbable que se llegue a mecanismos de defensa como la supresión o negación, con la consecuencia de un aprendizaje patológico que solo empeoraría la situación problemática $^{13}$. Por ello, es de gran

13 Para la problemática indicada vid. Senghaas Dieter, (2004) Zum irdischen Frieden. Erkenntnisse und Vermutungen, Frankfurt a.M. Suhrkamp, cap. 3. importancia que la ciencia contrarreste tal tendencia con contribuciones para un aprendizaje innovativo. Esto puede hacerse mediante juicios serios sobre la situación como el del Panel Intergubernamental del Cambio Climático (IPCC), o con perspectivas teóricas y prácticas constructivas de mediano y largo alcance, como lo hacen los discursos científicos y políticos sobre modelos del orden mundial con su correspondiente gobernanza mundial, derecho mundial interno y derecho mundial.

En vista de estos problemas reales a nivel mundial, estos esfuerzos desempeñarán un papel catalizador importante para la formación de una «esfera pública mundial» y permitirán superar tanto la tendencia a la autoreferencia de los discursos que se desenvuelven en cada disciplina como la tendencia al autismo de los discursos políticos cotidianos. Asimismo, permitirán contrarrestar las disparidades globales, al menos a nivel intelectual y analítico y ojalá también en el plano práctico-político: ¡una tarea centenaria! 